\title{
APLICACIONES DEL SISTEMA DE ESLABONES DE PUNI A LA PREPARACIÓN PSICOLÓGICA DE UN EQUIPO DE FÚTBOL ANTE COMPETICIONES CONCRETAS
}

\author{
Pedro Ureña Bonilla \\ Especialista en Psicología del Deporte \\ Universidad Nacional, Costa Rica \\ purena@medvet.una.ac.cr
}

RESUMEN

El sistema de eslabones propuesto por Puni se basa en una serie de pasos fundamentales en la preparación psicológica de los deportistas ante una competencia concreta. En primera instancia se hace referencia a: (a) la selección de información necesaria, suficiente y confiable acerca de las condiciones de la próxima competición y sobre las particularidades del rival, (b) al análisis de los recursos y posibilidades del propio equipo, (c) a la definición de estrategias de juego y de objetivos específicos individuales, (d) a la actualización de motivos personal y socialmente importantes en concordancia con los objetivos de la participación, (e) a la modelación de condiciones de la próxima competición, $(f)$ a la preparación especial para el encuentro, utilizando obstáculos competitivos de diversa gravedad (especialmente acontecimientos inesperados), $(g)$ a la definición de mecanismos de autorregulación de estados internos desfavorables y (h) a la selección y utilización de procedimientos de activación psicofísica precompetitiva óptima.

PALABRAS CLAVES: entrenamiento psicológico, preparación psicológica.

\section{APPLICATIONS OF PUNI'S LINK MODEL TO THE \\ PSYCHOLOGICAL PREPARATION OF A FOOTBALL TEAM BEFORE COMPETITION}

ABSTRACT

\begin{abstract}
The link model proposed by Puni is based on a number of key steps for the psychological preparation of athletes before competition. Reference is made to (a) the selection of necessary, sufficient, and reliable information regarding details of the next competition and the particularities of the opponent; $(b)$ analysis of the team's own resources and potential; (c) definition of game strategies and specific individual objectives; $(d)$ updating of personally and socially important motivations in accordance with competition objectives; $(e)$ modeling of the circumstances for the next competition; $(f)$ special preparation for the competition using obstacles with different levels of difficulty (particularly unexpected situations); $(g)$ definition of self regulatory mechanisms of unfavorable internal emotional states, and $(h)$ selection and use of techniques to trigger optimal psychophysical states before competition.
\end{abstract}

KEY WORDS: Psychological training, psychological preparation. 


\section{INTRODUCCION}

Sin duda alguna, gran parte del éxito deportivo depende de factores psicológicos (Srebro, 2002; Goldberg, 1997; Sonneschein, 2001; Eberspächer, 1995). De manera que, el entrenamiento moderno para la competencia, implica necesariamente la consideración de aspectos de orden mental y emocional (Ureña, 2003; Eriksson, 2001; Sonnenschein, 2001; González, 1996; Eberspächer, 1995). Sin embargo, además de los prejuicios con que ha tropezado la implementación del trabajo psicológico a nivel deportivo, la instrumentalización de los modelos teóricos propuestos no ha sido la más exitosa (Sonneschein, 2001). El punto de partida del entrenamiento psicológico en el caso de un equipo de fútbol, es contar con un proyecto de grupo en el que los jugadores tienen claridad en cuanto a las metas deportivas, las han interiorizado y se han comprometido con ellas. Es decir, se trata de una empresa en la que cada uno pone su talento al servicio de aspiraciones propias, colectivas, aceptadas implícita y explícitamente. Con este marco de entendimiento y de acuerdo con la propuesta de Puni (1969), la preparación psicológica de un equipo de fútbol ante un juego específico se podría llevar a cabo atendiendo los siguientes pasos:

\section{Paso 1: Búsqueda y selección de información sobre el rival: debe ser suficiente y confiable.}

Se trata de buscar por todos los medios necesarios, información fidedigna acerca del rival que se enfrentará. El espionaje y la observación de videos recientes sobre el adversario representan recursos de gran importancia. No se trata simplemente de ver cómo se mueve y juega el rival, sino más bien de analizar de manera pormenorizada, cuáles son las fortalezas y debilidades técnicas, físicas, tácticas y psicológicas que definen el perfil del futuro adversario. La participación analítica y crítica del grupo es fundamental. No solo se pondrá bajo la lupa el accionar general del equipo rival, sino que se estudiará línea por línea y puesto por puesto, lo que cada jugador hace y deja de hacer.

Para realizar el trabajo anterior, se sugiere dividir el grupo de jugadores en subgrupos y asignarles la tarea de analizar al rival a partir del siguiente esquema. El análisis será tanto del colectivo como de las individualidades.

a. Fortalezas :

- Físicas :

- Técnicas :

- Tácticas :

- Psicológicas :

b. Debilidades :

- Físicas :

- Técnicas :

- Tácticas :

- Psicológicas :

Se concluye con una sesión plenaria de análisis, discusión y conclusiones. Acto seguido, es importante tomar nota de los aportes concluyentes del grupo, a fin de tener elementos comparativos durante el proceso de revisión retrospectiva del juego. 
Se recomienda invertir máximo una hora en este ejercicio. Además, el punto central de este análisis es profundizar en el conocimiento y comprensión del adversario.

\section{Paso 2: Análisis de recursos y posibilidades del propio equipo}

Por supuesto que no basta con conocer las debilidades y fortalezas del rival. Es preciso también, tener conciencia absoluta de los recursos con que cuenta el equipo. El mismo esquema seguido en el análisis anterior, puede ser implementado en este segundo paso de la preparación. Obsérvese que hasta acá, se ha recurrido de manera particular a las posibilidades cognitivas de los jugadores, básicamente a la capacidad para pensar, es decir, a la destreza implicada en el análisis y síntesis de información (Sonneschein, 2001). Se podría incluso afirmar que, con estos ejercicios se induce la preparación mental del futbolista para el juego futuro (Eberspächer, 1995; Syer \& Connoly, 1987).

a. Fortalezas :

- Físicos :

- Técnicos :

- Tácticos :

- Psicológicos:

b. Debilidades :

- Físico :

- Técnico :

- Táctico :

- Psicológico :

En este momento del análisis, es fundamental concentrar la atención en el propio equipo, tanto desde la perspectiva de lo colectivo como a nivel individualizado. En consecuencia, el plan de entrenamiento en el terreno de juego será congruente con el diagnóstico realizado, con la filosofía de fútbol que se pretenda implementar y con la información recopilada sobre el adversario.

\section{Paso 3: Definición de estrategias de juego y de objetivos específicos}

Con base en la información obtenida en los pasos anteriores, se procede a hacer partícipes a los jugadores de la definición de aspectos básicos de una posible estrategia de juego contra el rival de turno. Entrenador y jugadores llegarán a un consenso sobre la propuesta de juego. Sin embargo, es preciso aclarar que, no se trata de suplantar la autoridad del entrenador, sino más bien de responder al carácter dinámico que tiene un equipo de fútbol y a la necesidad de consolidar el concepto de este. El entrenador obviamente será quien tome las decisiones finales. De manera que, la globalidad de la propuesta de estrategia de juego se concibe entre los actores involucrados: jugadores y cuerpo técnico.

Es indispensable en este punto la definición de metas colectivas e individuales para el juego, ya que ello es de gran importancia psicológica (Srebro, 2002; Eriksson, 2001; Cabrini, 1999; González, 1996). Entre las razones que con mayor frecuencia se mencionan están: a) el trabajo con metas u objetivos, estrategia que le ayuda al futbolista a concentrar y canalizar su energía, a regular el esfuerzo; por otro lado, le motiva a seguir luchando, en consecuencia, promueve la persistencia, la resistencia mental y la voluntad. Asimismo, hace que el futbolista tenga que ocuparse de aquellas áreas en que necesita mejorar, orientando el trabajo individualizado. Además, le permite al futbolista ganar confianza, seguridad y convicción, factores decisivos 
para el logro de rendimientos óptimos. Finalmente, le permite a este y al cuerpo técnico valorar de manera objetiva partido a partido, cuál es el aporte real del jugador, al menos en la dimensión de lo cuantificable. A partir del sojuzgamiento de los rendimientos objetivos, se podrá inferir la capacidad psicológica de rendimiento del futbolista.

En relación con el uso de metas, Locke \& Latham (1991) llegaron a las siguientes conclusiones:

1. Se debe trabajar con metas específicas, por cuanto éstas hacen que la actividad sea más efectiva.

2. Las metas que suponen un reto hacen que el rendimiento sea mayor que en el caso de metas fáciles.

3. El logro de metas a corto plazo son el camino para el logro de las de largo plazo.

4. El establecimiento de metas mejora el rendimiento del deportista.

5. El trabajo con metas solo funciona si existe retroalimentación oportuna.

6. Las metas deben ser aceptadas por el deportista para que sean efectivas.

7. La consecución de metas se facilita si se tienen planes de acción o estrategias adecuadas.

De manera que, es tarea del cuerpo técnico apoyar y orientar al futbolista en la definición de ellas, darle seguimiento al cumplimiento de las mismas y retroalimentar al deportista, para finalmente proponer situaciones de entrenamiento tanto individuales como colectivas que lo preparen para el logro de las metas establecidas.

Ejemplo de metas colectivas:

1. No permitir un solo remate a marco en los primeros 10 minutos

2. Quitarle el balón al rival en su propia media cancha al menos 10 veces en el primer medio tiempo.

3. Otras

Entre algunas posibles metas individuales se tienen:

1. Realizar al menos 10 anticipaciones durante el partido.

2. Provocar al menos 5 tiros de esquina a favor.

3. Lograr al menos 2 buenos remates durante el partido.

4. Realizar al menos 1 asistencia para gol.

La idea fundamental es que, una vez definida, aunque a grandes rasgos la estrategia de juego a utilizar con el rival de turno, se proceda a plantear con los jugadores la metas particulares. Por supuesto, no bastará con establecerlas a nivel teórico, sino que se deberá preparar situaciones de entrenamiento que en el marco de la propuesta estratégica, enfrenten al futbolista con la necesidad de logro de las metas particulares establecidas. Será tarea del cuerpo técnico, en particular del entrenador conjuntamente con el asesor en el campo psicológico deportivo, velar porque se trabaje intensamente en función de las metas propuestas. Cuando el jugador llegue a la situación real de juego, contará con muchos más recursos mentales para cumplir de manera eficiente y eficaz su rol.

Será tarea del entrenador o en su defecto del asesor en el campo psicológico deportivo, o de ambos, diseñar boletas de metas específicas para cada jugador. De la misma forma, les corresponderá evaluar el logro de las mismas, retroalimentando a los jugador y repetimos, creando situaciones de entrenamiento que preparen al futbolista para el logro respectivo de las mismas. De tal forma que, se tendrá que realizar sesiones individuales de trabajo para identificar y concretar metas. 
En todo caso, es sano y recomendable que al inicio de toda sesión de entrenamiento, el entrenador exponga ante el grupo, cuáles serán los objetivos del trabajo, espacio que se aprovechará para mencionar no solo metas colectivas, sino también, para llamar la atención sobre la importancia de los objetivos individuales que cada quien deberá cumplir y de las situaciones de juego que se crearán para el logro de los mismos (Sonneschein, 2001).

Tabla 1. Ejemplo de boleta con definición de metas individuales

Nombre del jugador:

Posición:

1. Realizar al menos 10 anticipaciones durante el juego

2. Provocar al menos 3 tiros libres cerca del área rival

3. Provocar al menos 2 tiros de esquina.

4. Hacer al menos 3 buenos remates a marco.

5. Realizar al menos 1 asistencia para gol

6. Otras

Bajo ninguna circunstancia olvidar el papel que tiene la retroalimentación. Es preciso que el cuerpo técnico brinde permanentemente información al futbolista después de cada actuación de manera que éste pueda corregir y mejorar. El futbolista debe saber que su actuación está siendo evaluada de manera constante, fundamentalmente con la intención de retroalimentar su rendimiento y mejorarlo.

\section{Paso 4: Actualización de motivos}

Cuando se habla de motivos, se está haciendo referencia a las razones que tiene el individuo para entregarse a la práctica del fútbol de manera profesional. Aquí se encuentra motivos de orden tanto exógeno como endógeno (Roberts, 1992, Duda, 1992, Nicholls, 1992). En el primero de los casos, se tendría aquellos futbolistas que juegan en razón de una buena presencia de público, de los premios que les ofrezcan, del reconocimiento que reciban en los periódicos, en la radio, en la televisión. Se trata de razones que están fuera del futbolista mismo. En el segundo de los casos, el orgullo, el disfrute del reto, la competencia, y el deseo de logro, se convierten en las razones más importantes para entregarse durante un juego. Este tipo de motivos son de naturaleza interna $\mathrm{y}$, sin lugar a dudas, son los que brindan el marco para el logro de rendimientos máximos.

Cuando se trabaja con metas orientadas hacia el proceso, tal y como se presenta en el paso 3, se está fortaleciendo y consolidando los motivos de naturaleza interna en el futbolista. En todo caso, se trata de despertar e intensificar el interés para actuar de una manera concreta en el partido específico. A la par del trabajo con metas, la definición de consignas de equipo, con el objetivo de energizar la acción del futbolista puede ser de gran utilidad. 
Ejemplo de consignas de equipo podrían ser:

1. No darse por vencido jamás durante el juego. Nunca dejar caer los brazos.

2. Mantener la concentración en lo que se tiene que hacer y no en lo que se debió hacer.

3. Motivarse y reforzarse permanentemente, empujándose cada vez a un esfuerzo mayor.

4. Apoyar y motivar a los compañeros de equipo de manera permanente.

5. Nunca dar una bola por perdida.

6. Nunca meterse con el árbitro.

7. De ninguna manera atacar al rival ni verbal ni físicamente.

8. Mantener una actitud positiva hasta el último minuto de juego.

9. Luchar hasta el final por la (s) metas propuestas.

El valor de estas consignas puede hacerse operativo diseñando situaciones de juego en que se destaque el contenido de cada una de ellas. Día a día se debe reflexionar sobre el valor de las mismas. Es preciso recordar que solo la repetición permite la maestría y la perfección.

En el proceso de actualización de motivos es preciso conocer las razones que mueven al futbolista. Una conversación profunda ayudará mucho a complementar la información necesaria para formar una idea más o menos precisa de la estructura motivacional del futbolista. Por supuesto, complementará esta información, la observación diaria del su comportamiento en el camerino, en el entrenamiento de campo, en las sesiones de trabajo teórico, y en los partidos (Sonneschein, 2001). Obviamente la sistematización de toda esta información es un trabajo delicado, pero que al final rinde sus buenos frutos. Al menos se tendrá futbolistas más autoconcientes y con una orientación hacia el logro mucho más clara.

Toda situación que estimule la entrega, el orgullo personal, el deseo de logro, entre otros motivos, significa una actualización de motivos, es decir, un retomar niveles de conciencia óptimos para entregarse por la causa del equipo (Gabler, 2004). Charlas, conversaciones personales, videos (el equipo recibiendo un premio específico, una condecoración, un reconocimiento) situaciones de juego, entre otras, podrían concebirse para despertar las razones realmente valederas para entregarse con todo a la práctica del fútbol.

\section{Paso 5. Modelación de condiciones}

En razón de lo analizado hasta el momento respecto del rival y del propio equipo, crear en los entrenamientos, situaciones de juego que simulen circunstancias posibles en el juego próximo (Eberspächer, 1995). Se trata de anticipar a partir de toda la información ganada, escenarios posibles, ensayarlos, vivirlos, superarlos.

Un concepto que puede ser de gran utilidad en este momento, al igual que en otros, durante el proceso de preparación específica es la visualización. Se trata de un trabajo complementario al entrenamiento de campo de gran utilidad (Srebro, 2002), con el que se involucra de manera fundamental la capacidad imaginativa del futbolista. Consiste en la habilidad para ver, escuchar y sentir las acciones propias, como en una pantalla dentro de la mente. Durante el proceso de la visualización el futbolista puede ejecutar diferentes acciones que le ayudarán a alcanzar sus metas. Expuesto de manera simple, es un proceso de pensamiento en el que se memorizan imágenes y sensaciones.

La capacidad para visualizar la tienen todos los seres humanos, sin embargo, no todos la usan de la mejor manera. No se trata de un sueño o fantasía y su utilización adecuada podría contribuir significativamente a mejorar el rendimiento del deportista. Al igual que cualquier otra destreza, la visualización tiene que ser aprendida y practicada. 
La visualización puede ser utilizada en diferentes circunstancias:

- Para mejorar la técnica personal.

- Para adquirir nuevas habilidades.

- Para comprender mejor el juego.

- Para fortalecer nuestra confianza y creencias acerca de nuestras habilidades y destrezas.

- Para mejorar el juego del equipo.

- Como complemento del entrenamiento para cambiar condiciones de juego.

- Para acelerar los procesos de recuperación pos lesión;

- entre otras.

Durante el proceso de la visualización se utilizan todos los sentidos, pero especialmente el sentido del movimiento. De manera que, entre más sentidos se vean involucrados en el proceso, más real será la experiencia y consecuentemente más efectiva.

La visualización ayuda a reducir la incertidumbre. Por medio de ella se fortalecen creencias de autoeficacia (Sonnenschein, 2001; Eberspächer, 1995). La imaginación se utiliza para anticipar de manera visualizada los diferentes momentos del juego. Así, cuando el futbolista está en una situación real en el, puede manejarla con mucha mayor propiedad.

El efecto de la visualización, es la mayoría de las veces, una sensación de bienestar, satisfacción y seguridad.

La hipótesis básica que da sustento a la utilización de la visualización como complemento del entrenamiento de campo es que, lo que el futbolista repase en su mente, en su imaginación, influirá directa y decisivamente en su rendimiento.

Es importante considerar los siguientes principios para hacer sacar el máximo provecho a esta destreza:

1- La persona es la productora de la película. Debe escoger una situación de juego que le interese de manera particular.

2- La visualización corta (menos de un minuto) puede hacerse en el campo sin necesidad de inducir relajación.

3- La visualización larga (más de 5 minutos) se ejecuta a partir de estados de relajación. Esto significa que se debe empezar con un ejercicio de relajación.

4- La visualización se hace con los ojos cerrados y en posición cómoda.

5- No trabaje la visualización por más de 3 a 5 minutos. Después de la visualización revertir el efecto de la relajación, utilizando fórmulas activadoras.

6- La visualización se hace siempre en el presente. En otras palabras la persona se dice a sí mismo... Yo estoy en el juego, esto está sucediendo en este momento.

7- Durante la visualización se practican acciones específicas. Cada ejercicio de visualización debe tener su meta particular.

8- La ejecución de sus acciones a nivel imaginativo, debe coincidir con el tiempo real que requeriría dicha ejecución, no en cámara lenta.

9- No imaginar cosas imposibles. Utilizar como parámetro el rendimiento propio, pero siempre aspirando a mejorarlo.

10- El ejercicio de visualización debe ser siempre de éxito.

11- Asegúrese de ejecutar sus acciones de manera correcta durante la visualización.

12- Entre más sentidos involucre en el proceso de visualización mucho más efectiva será ésta. 
Hay dos formas de llevar a cabo la visualización: Una desde afuera, es decir, el deportista se observa a sí mismo como en televisión, con la cámara siguiéndolo. Dos, desde adentro, o sea, el deportista ve el balón o la meta como si tuviese una cámara en la cabeza.

13- Mientras visualiza, se debe estar tan alerta, como cuando ejecuta realmente la acción.

14- La visualización no es un sustituto del entrenamiento en el campo, si un complemento psicológico indispensable para el logro de rendimientos máximos.

Al introducir el trabajo de la visualización, se está promoviendo en los jugadores el juego mental anticipado de situaciones concretas de juego y el mismo.

\section{Paso 6. Preparación especial para el encuentro utilizando obstáculos competitivos (especialmente inesperados).}

La utilización de obstáculos es un complemento de la modelación de las condiciones de competencia. Consiste en el uso de situaciones de juego, ya sea favorable o desfavorable que obliguen al jugador o al equipo, a replantear y ajustar esquemas tácticos o bien, actitudes ante circunstancias de juego que podrían tener un peso psicológico importante para el rendimiento del futbolista. Por ejemplo, se recibe la expulsión de uno o dos jugadores, se juega con uno o dos jugadores más que el rival, se tiene el marcador en contra en los últimos 10 minutos del segundo tiempo, entre otras.

En este contexto, la utilización de la visualización podría ser de gran utilidad para estimular procesos de adaptación óptimos ante situaciones "inesperadas" durante un partido. Otra posibilidad de preparación ante este tipo de situaciones la brinda el entrenamiento de situaciones irrepetibles (Eberspächer, 1995). Aquí, se trata diseñar situaciones únicas, por ejemplo, un tiro de esquina, un tiro libre, un lanzamiento de penal. En esencia se trata de forzar a que la atención del equipo se focalice en la realización de esa acción, la que será ejecutada una única vez durante el entrenamiento. Introducir condiciones alrededor de la situación en cuestión, por ejemplo, se ubica en los últimos diez minutos del juego futuro, se pierde por un gol, o se gana por un gol, entre otros posibles ingredientes. Nuevamente, la capacidad de los futbolistas para vivenciar de manera intensa tales circunstancias de juego es fundamental. El éxito de este tipo de entrenamiento depende de la seriedad con que los futbolistas asuman estos retos. Por supuesto que, el entrenador o en su defecto el asistente o asesor psicológico deportivo debe llevar un registro de ejecuciones y logros. De manera que se pueda retroalimentar los procesos de corrección y perfeccionamiento en la ejecución de situaciones estándar o cualquiera otra.

\section{Paso 7. Definición de mecanismos de autorregulación de estados internos desfavorables}

Este paso consiste en la identificación de aquellas sensaciones y estados del futbolista que puedan ser negativas para su rendimiento, y consecuentemente para el logro de las metas del equipo. Por otro lado, se pretende identificar mecanismos de autorregulación utilizados por el , valorar conjuntamente la eficacia de los mismos y replantear en lo necesario y posible otras opciones de autocontrol (Srebro, 2002).

La conversación abierta con el futbolista, así como la observación minuciosa de su comportamiento en situaciones de entrenamiento y de juego, serán fundamentales para obtener información sobre los mecanismos de regulación que utiliza.

Una de las técnicas a que puede echar mano el futbolista es la detención de pensamientos. Aquí se requiere que el futbolista desarrolle el hábito de registrar sus pensamientos negativos (hoy no 
será mi día, hoy me siento flojo) y responder esos pensamientos con fórmulas alternativas positivas. La idea es que el futbolista aprenda a sustituir pensamientos negativos por positivos apenas aparezcan (Srebro, 2002). Se requiere en primera instancia convencer al futbolista para que auto investigue sobre sus propios patrones de pensamiento en situación de entrenamiento o competitiva. A fin de dominar el uso de esta técnica se recomienda seguir los siguientes pasos:

- Aprenda a imaginar efectivamente

- Identifique las situaciones ante las cuales se activan diálogos internos negativos

- Identifique pensamientos negativos específicos

- Identifique una señal que permita bloquear el pensamiento negativo

- Haga una lista de afirmaciones positivas que reemplacen a las negativas.

Es importante tener presente que, el proceso de bloqueo de pensamientos requiere desarrollar la capacidad imaginativa.

Otra de las técnicas que podría ser de gran utilidad es la denominada "El Pensamiento Racional" (Ellis cit. en Martens, 1987). La técnica se basa en el modelo ABC descriptivo del fenómeno de la vivencia de estrés. Donde $\mathrm{A}=$ Activación de evento (s) o situación ( $\mathrm{s}$ ), $\mathrm{B}=$ Convicciones o percepciones sobre la situación y $\mathrm{C}=$ Consecuencias de la situación.

La hipótesis fundamental es que, las creencias sobre las situaciones son las que generan la pérdida de control.

El primer paso en la técnica es, identificar los pensamientos irracionales y negativos. Estos pensamientos pueden ser divididos en cuatro categorías: a) tensión a propósito del rendimiento, especialmente como anticipación del propio rendimiento en comparación con el de otros; b) preocupación a causa de los síntomas físicos asociados con la vivencia de estrés; c) reflexiones acerca de las posibles consecuencias negativas de un mal rendimiento, incluido, el castigo, la pérdida de autovalía, la desaprobación y la reducción de oportunidades para el futuro y, d) pensamientos relacionados con ineficacia, los cuales pueden incluir la autocrítica y la autoofensa.

En este caso, el futbolista debe ser motivado a hacer una lista de los pensamientos negativos propios y a identificar los más comunes. Al concluir este paso, en lo sucesivo se indagará sobre la causa de los mismos y se determinará cuáles están dentro del rango de control personal y cuáles no. Posteriormente se buscan razonamientos lógicos y se proponen pensamientos alternativos positivos sustitutivos. Al fin de cuentas, se trata de ayudarle al futbolista a construir un plan de afrontamiento que lo conduzca al despliegue máximo de su talento.

\section{Paso 8. La selección y utilización de procedimientos de activación psicofísica óptima precompetitiva.}

El logro de estados de activación psicofísica óptimos es fundamental para el logro de rendimientos máximos. Al respecto, cada futbolista debe desarrollar su propia técnica de movilización.

Eberspächer (1995), distingue tres puntos de apoyo en los que podría orientarse el desarrollo de un procedimiento de activación o movilización, a decir, el comportamiento, el entorno y la percepción. En el primero de los casos, se trata de inducir movimientos rápidos y explosivos. Utilizar ejercicios isométricos que aumenten el tono muscular. En relación con el entorno, se debe buscar y promover una atmósfera excitante. Y finalmente, en cuanto a la percepción, se trata de predisponerse por medio de la auto exigencia y la auto-conversación. 
La utilización de videos cortos, con jugadas espectaculares terminadas en gol, representan una alternativa de gran utilidad para generar la sensación de éxito y alcanzar estados de movilización óptimos.

\section{Conclusiones:}

Aunque de hecho, el factor psicológico está presente en todas las sesiones de entrenamiento de los equipos de fútbol, los niveles de conciencia y la sistematicidad con que se atienden, son realmente deficitarios. La serie de pasos expuestos en la preparación psicológica de un equipo fútbol, antes de un juego, representa la esquematización de una serie de posibilidades que le podría permitir a los cuerpos técnicos reducir niveles de incertidumbre, al atender un número mayor de los factores decisivos en el logro del éxito deportivo. A lo largo de los eslabones propuestos se destaca el valor que tiene el enfrentamiento cognitivo con las situaciones propias de la competencia deportiva, de manera que, el trabajo cara a cara con el futbolista, representa, quizá, la mejor opción para el desarrollo de las posibilidades psicológicas del mismo.

\section{Referencias bibliográficas}

Cabrini, M. (1999). The Psychology of Soccer. Pennsylvania: Reedswain.

Duda, J. (1992). Motivation in Sport Settings: A Goal Perspective Approach. In G. Roberts (Ed.), Motivation in Sport and Exercise (p. 57-93). Champaign, Il.: Human Kinetics.

Ebärspecher, H. (1995). Entrenamiento mental: Un manual para entrenadores y deportistas. Barcelona: INDE.

Eriksson, S-G. (1997). The Inner Game - Improving Performance on Football. Italy: Carlton Book.

Gabler, H. (2004). Motivationale Aspekte Sportlicher Handlungen. In H. Gabler, J. Nitsch \& R. Singer (Hrsg.), Einführung in die Sportpsychologie (s.197-244). Schorndorf: Hofmann.

Goldberg, A. (2001). Playing out of you mind: A soccer player and coach's guide to developing mental toughness. Auburn, Michigan: Reedswain Publishing.

González, J. L. (1996). El entrenamiento psicológico en los deportes. Barcelona: Biblioteca Nueva.

Martens, R. (1987). Coaches Guide to Sport Psychology. Champaign- Illinois: Mouvement Publication.

Nicholls, J. (1992). The General and Specific in the Development and Expression of Achievement Motivation. In G. Roberts (Ed.), Motivation in Sport and Exercise (p. 3157). Champaign, Il.: Human Kinetics.

Locke, E. \& Latham, G. (1991). Establecimiento de objetivos en el deporte (págs. 140-165). En J. Riera \& J. Cruz (Eds.), Psicología del Deporte: Aplicaciones y perspectivas. Barcelona: Martínez roca.

Puni, A.Z. (1969). La preparación psicológica para las competiciones deportivas. Habana, Cuba: INDER. 
Roberts, G. (1992). Motivation in Sport and Exercise: Conceptual Constraints and Convergence. In G. Roberts (Ed.), Motivation in Sport and Exercise (p.3-31). Champaign, Il.: Human Kinetics.

Sonneschein, I. (2001). Training psychischer Handlungsvoraussetzungen im Leistungssport (s. 85-3). In H. Gabler, J. Nitsch \& R. Singer (Hrs.), Einführung in die Sportpsychologie. Schorndorf: Hofmann.

Srebro, R. (2002). Winning with your head. A complete mental training guide for soccer. Auburn, Michigan: Reedswain Publishing.

Ureña, P. (2003). El entrenamiento psicológico en el deporte de alto rendimiento (págs. 85-93). Revista ABRA, Universidad Nacional, Heredia: EUNA.

Fecha de recepción del artículo: marzo del 2005.

Fecha de aceptación del artículo: julio del 2005.

Fecha de publicación del artículo: 26 de julio del 2005. 